\title{
The Role of Mathematical Literacy in the Artistic Design of Floral Jelly Cakes
}

\author{
Tsui-Ying Huang1, Ting-Sheng Weng2*, Yang Chen ${ }^{3}$ \\ ${ }^{1}$ Department of Bio-Business Management, NCYU, Taiwan \\ ${ }^{2}$ Department of Business Administration, NCYU, Taiwan \\ ${ }^{3}$ Executive MBA Program at College of Management, NCYU, Taiwan \\ Email: tyhuang2882@gmail.com, *politeweng@mail.ncyu.edu.tw, You0983811373@gmail.com
}

How to cite this paper: Huang, T.-Y., Weng, T.-S., \& Chen, Y. (2021). The Role of Mathematical Literacy in the Artistic Design of Floral Jelly Cakes. Open Journal of Social Sciences, 9, 219-231.

https://doi.org/10.4236/jss.2021.99016

Received: August 13, 2021

Accepted: September 10, 2021

Published: September 13, 2021

Copyright $\odot 2021$ by author(s) and Scientific Research Publishing Inc. This work is licensed under the Creative Commons Attribution International License (CC BY 4.0).

http://creativecommons.org/licenses/by/4.0/

\begin{abstract}
Although artistic and delicate shapes can be made in creative design teaching on site, standard sizes should be specified for some necessary types of objects, in order to provide guidance for students and teach them techniques. It can be found that many technical tutors fail to teach students the quantitative characteristics of mathematics in making a jelly flower. As a result, it is difficult for novices to select accurate types of flower needles, and to know that different flower buds and petals should be created by injecting liquid concoctions with different and precise angles, depths, and sizes. Petals in different lengths should also be designed for tutors to teach students techniques with precise information. As no flower needles or concoction ratios similar to those mentioned above are provided in films, teaching files, or books, it is necessary to teach the flower piping with different angles, lengths, depths. This study aims to provide students with accurate numbers and angles that follow the technical steps and methods to design and make delicate floral jelly cakes, as well as the use of various quantitative characteristics with explanations through graphs and text. The results of this study provide novices with the required techniques to make these cakes. This study can help tutors learn from examples and engage in teaching suitable and scientific techniques, and its result can be taken as the basis for the development of a professional teaching course in making floral jelly cakes.
\end{abstract}

\section{Keywords}

Floral Jelly Cake, Mathematics Literacy, Mathematics and Art, Technical Teaching, Educational Technology 


\section{Introduction}

With the popularity of bakery chains, coffee and cake shops, and restaurant chains, gourmet cakes have become part of most people's diet. Different cake ingredients have an influence on the design of shapes, and the various combinations of taste and appearance attract more consumers. Chewy floral jelly cakes are flavored with diverse tastes (Juan et al., 1995), and thickeners are used for jelly solidification, including gelatine, agar-agar, gelatine sheets, mousse powder, jelly powder, and pudding powder (Ishibashi, 2020). Jelly powder in Taiwan is approximately composed of agar and Tacca.

Bian (2018) mentioned that cake shapes can make floral jelly cakes more pleasing when in accordance with the customization requirements of mathematical literacy. Due to the growing popularity and public awareness of this cake, its appearance and artistic shape have changed. As vision affects taste, a small change in the shape design can bring surprises when people taste the cake, and a soothing and aesthetic cake will delight people.

\section{Motivation}

Huang and Chou (2015) mentioned that more attention is given to taste and smell by cake makers to satisfy customers, rather than vision and touch in design. Due to the differences in material and quantities in the initial preparation, as well as the visual aesthetics of the internal and external shapes in the final process, mathematical literacy is indispensable in the design and production of this cake. Artistic desserts must be designed, processed, and produced using diverse ingredients, cooking steps, and time control. Cakes in artistic forms bring satisfaction to individuals when they are enjoying the desserts. Ingredient preparation is a mathematical process, including quality and quantity, order and time, and techniques, such as cooking, sculpturing, and processing.

Cake practitioners need to keep pace with the times and changing technologies, such as cake production with artificial intelligence machines and new methodologies, and thus, must develop key mathematical and artistic literacy.

\section{Purpose}

Based on the requirements of modern people regarding the color and flavor of cakes, ingredient proportions can result in different colors and flavors. Therefore, this study introduces mathematical literacy into the practice of design teaching, and applies the mathematical concepts into the structure of floral jelly cakes from the point, line, and surface perspectives, in order to complete these artistic cakes and enrich the studies on the techniques.

When cake makers have artistic and mathematical literacy, they can teach students the complete system in this field, including application of the correct mathematical design techniques when making the cake, and teaching aesthetics and appearance as a way to develop students' ability in making the required artistic shapes, which integrate mathematics and art. Moreover, teachers can provide a 
more lively and diversified teaching method. For the cake sellers, these diverse and artistic works are bound to attract more customers and gain popularity.

This study aims to systematically sort out the production process of floral jelly cakes through practical operations for technical teaching. It is expected that the results of this study can facilitate the entrepreneurial spirit of positive innovation and flexibility, and technical education can be achieved by training cake-making talents. This study tries to stimulate students' interest in mathematical literacy to demonstrate the spatial art of the cake works and make this cake a piece of spiritual art and a delicious feast.

\section{Literature Review}

\subsection{Visual and Aesthetic Art}

Yu (2016) mentioned in the book The Story of Seeing Beauty: "From the primitive human murals, the shapes of implements, accessories and decorations, all remains the expression of beauty. Beautiful characters in all kinds of language prove that the consciousness of beauty has existed long before."

$\mathrm{Lu}$ (1984) suggested that shape (Xing Zhuang) is one of the basic conditions for humans to perceive an object. Xing refers to the appearance of an object, while Zhuang refers to the state. When objects are formed, different materials, shapes, and colors suitable for fusion are required to stimulate the visual perception of individuals and generate aesthetic perceptions through images, forms, and elements (Chuang \& Chen, 2004). Chang (1988) mentioned that form as a formal expression is indispensable for the composition of products. This study takes a cake with abundant shapes as an example. While the basic forms of cakes are determined by different diets and cultures, cakes mainly remain circular columns. This study focuses on the design of jelly cakes, which are transparent and gelatinous. When the shape of an object is generated in the vision of people, the information constructed in the picture will be combined and classified in different shapes; for example, different objects may be formed by straight or curved lines in cake designs to have visual significance to the spatial shape of the cake, including straight lines, geometric shapes, such as squares, triangles, and polygons, and curves, such as circles, ovals, and curves. However, Hsiung, Chu, and Chang (2005) mentioned that form (Xing Tai) generally refers to conveying the meaning of the object itself. Xing represents the appearance and shape of the object, and Tai refers to its inner spirit. In the field of design, the form of a product is mainly composed of abstract geometric forms and bionic forms and is conceived in accordance with the content of the product, mainly from the product. Nevertheless, the majority of consumers are usually attracted by the appearance of the product. This study focuses on the appearance, perspective, and dynamic characteristics of transparent floral jelly cakes, thus, the internal shape of the cake is very important for the design. The design spirit of the dynamic aesthetics inside the cake will also have an effect on consumers' purchase intention. 
Chen (2008) discussed the creative intention of artists and the real meanings conveyed by images. This study suggests that for different cake designers, their dynamic and spatial cake designs will have similarities and differences. In addition to imitating the techniques of tutors, how can students stimulate their passion and understanding of creative design? Students in cake design classes should continuously give play to their creativity in designing dynamic cake images with limited space, in order to present their perspective images with artistic and visual perceptions, thus, it is necessary to carry out and forward the integration of mathematics into art and technology education.

\subsection{The Influence of Mathematical Literacy on Artistic Objects}

Regarding the connection between mathematical knowledge and life, there must be an object playing a bridging role, such as "mathematical literacy" (Tso \& Lei, 2018). In the past, mathematical teaching attached great importance to the teaching of knowledge and abilities. However, the issues that require integrating abilities in solving problems cannot be smoothly resolved using mathematical knowledge. In particular, this challenge is posed by the lack of mathematical literacy (Cheng, Tseng, \& Chao, 2020).

Mathematical literacy is defined in the mathematical assessment framework of PISA (Programme for International Student Assessment) 2022 (PISA National Center, National Taiwan Normal University, 2021) as "the ability of individuals to make mathematical reasoning in a variety of real-world contexts, and to develop, apply, and interpret mathematics to solve problems, including the use of mathematical concepts, procedures, facts, and tools to describe, explain, and predict phenomena. Mathematical literacy promotes the individual understanding of the role mathematics plays in the world, and it enables individuals to make informed judgments and decisions, becoming what the citizens in the 21st century need with construction, input, reflection (OECD, 2018)." An important concept of mathematical literacy is to use mathematics to solve problems in life, which includes personal and career-related aspects, as mentioned in PISA. The required mathematics abilities include quantity, uncertainties in data, change and relation, space, and shape, which strongly require quantification of the characteristics and properties of all concepts, such as size, quantity, length, and weight. By understanding the characteristics of quantification, surroundings can be solved on the basis of those quantities. Making floral jelly cake requires makers to grasp the above quantified characteristics in order that the necessary dynamic flower shapes can be designed and formed within the limited jelly space. Therefore, it is necessary for technical educators in vocational training to inculcate and teach students quantitative characteristics.

Ouyang (1994) proposed that the interest and great vitality of mathematics lies in its enlightenment to thinking. Mathematics is the gymnastics of thinking (Huang, 1994) and a key factor in cultivating quality students. Based on guiding students to discover and explore mathematical knowledge and concepts, the fu- 
sion of mathematics and art results in mutually integrated knowledge, the communication of ideas, and innovative thinking. In this way, students can deeply feel and experience mathematics through the charm of art, see mathematical knowledge applied in art, enjoy mathematics from the artistic perspective, and understand the initial literacy of art from the perspective of mathematics. The integration of mathematics and art not only demonstrates rich mathematical knowledge, ideas, and methods, it also communicates the mathematical culture (Xiong \& Zhang, 2015). Tseng (2000) stated that mathematics creates the knowledge of human science, and it is the art of thinking where mathematics itself is the art. Artworks can beautify the spirits of people. Yi and Wang (2007) mentioned that mathematics is an important component of culture in life through aesthetic art (Liu, 2016). Zhang (2007) discussed the role of mathematics in art, and from the understanding of mathematics and the influence of people's disposition, found that it enlightens educational reform and artistic literacy, and creates high caliber talents. This study suggests that through practice, mathematical truths can be experienced to improve creativity. Therefore, mathematical literacy is considered a bridging object that would inspire people to incorporate mathematical knowledge with other fields.

\section{Procedure and Analysis}

\subsection{Techniques for Making Floral Jelly}

The relationship between the technique and mathematics of making floral jelly cake is illustrated, as follows: Squeeze the plunger gently until a small ball is pushed out, slowly withdraw the needle to make an elongated petal shape, then release the pressure and remove the needle.

1) First prepare tools and materials (Figures 1-4).

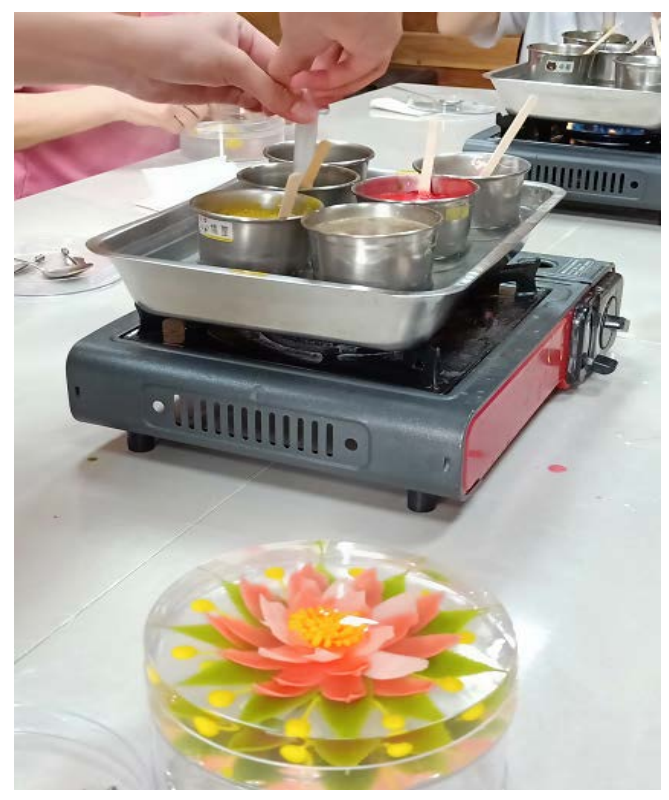

Figure 1. Boiling floral jelly liquid and floral jelly cake. 


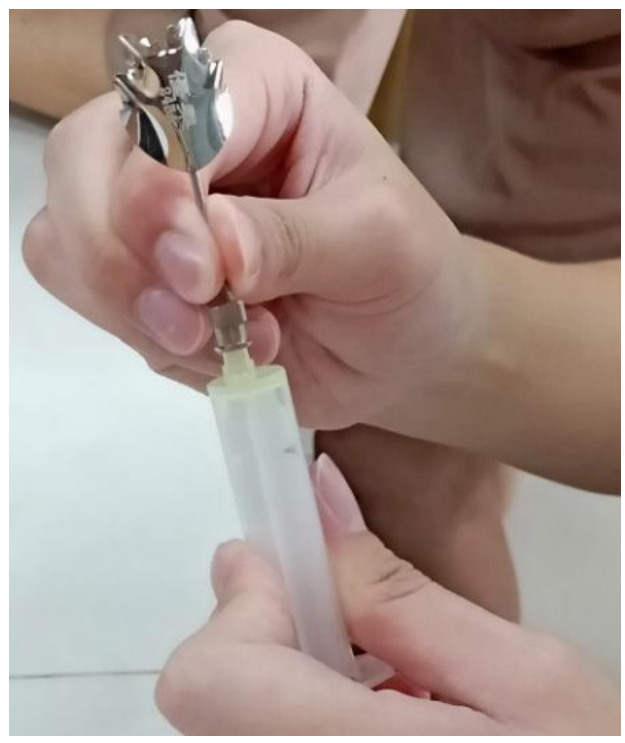

Figure 2. A shaping needle.

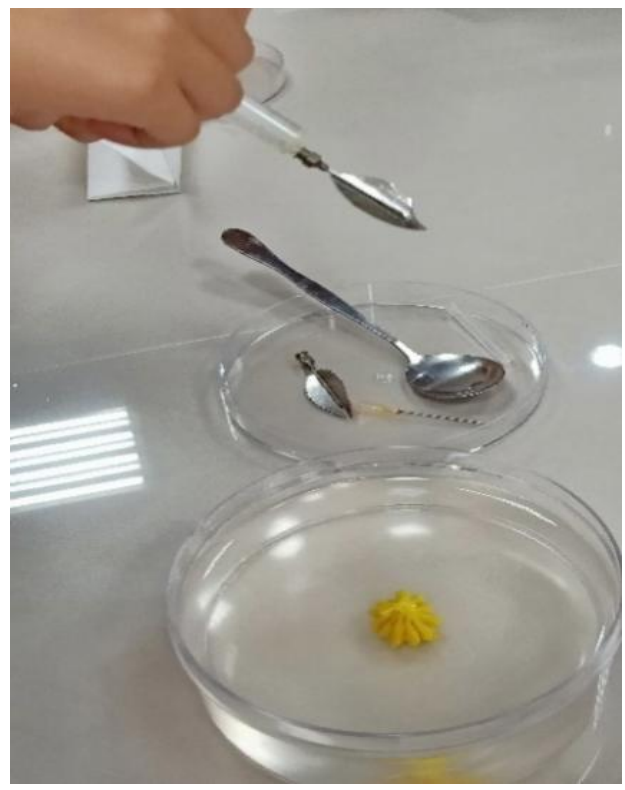

Figure 3. Shaping needles and jelly bud.

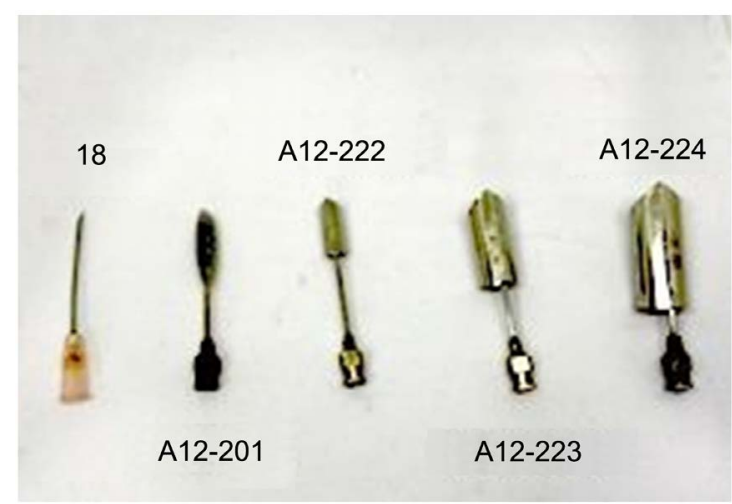

Figure 4. Types of tools. 
2) Measure konjac powder $280 \mathrm{~g}$, white granulated sugar $600 \mathrm{~g}$, and water $5000 \mathrm{~g}$. Put the mix of $280 \mathrm{~g}$ konjac powder and $600 \mathrm{~g}$ white granulated sugar in a container and stir well. Pour in $5000 \mathrm{~g}$ water and soak for 10 minutes. Bring to a boil over high heat, lower the heat and cook for 3 to 5 minutes until it becomes transparent, and turn off the heat. Cool for 20 to 30 minutes, remove the film on the transparent jelly, then pour it into the prepared container to complete the transparent jelly.

3) The colors of the leaves and flowers to be used in the jelly should also be prepared in order and in accordance with the weight in grams and the time required.

As can be seen from the above, mathematics is applied everywhere to make the floral jelly, such as different materials with different weights and different times for boiling and cooling.

The following instructions design stamens, petals, and leaves as examples:

4) Taking the design of the flower stamen as an example (Figures 5-7): Use the 18-gauge needle (Figure 4).

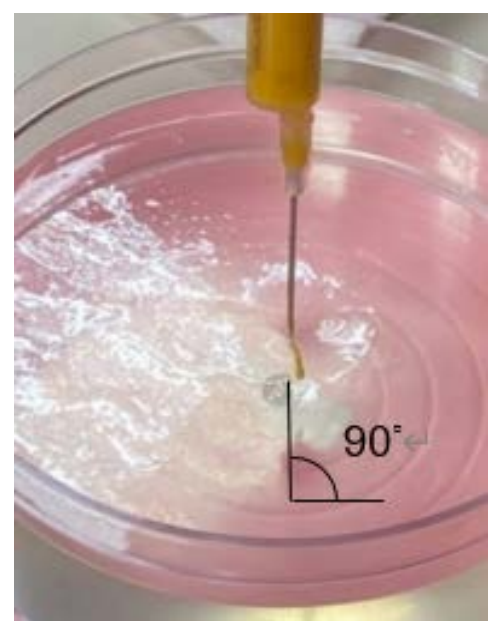

Figure 5. Direct injection at 90 degrees.

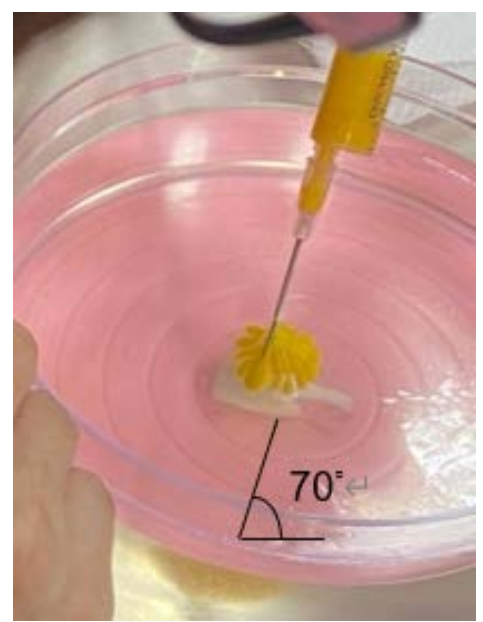

Figure 6. Injection at 70 degrees. 


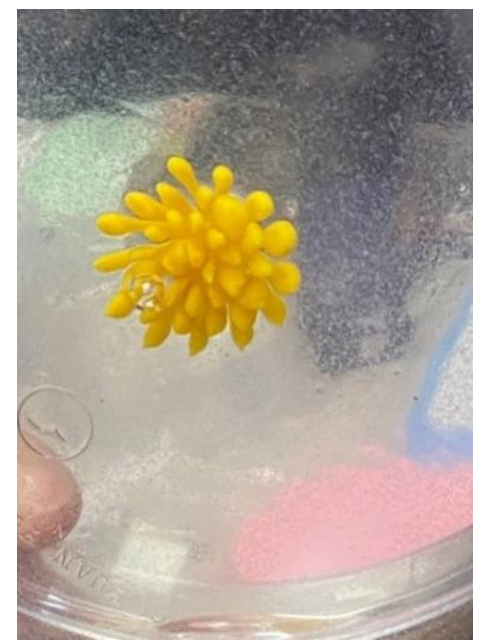

Figure 7. Turn to the front to see the shape of bud.

a) Using an 18-gauge needle, fill the cylinder with yellow liquid jelly, as shown in Figure 5. The first needle is injected in the 90-degree vertical direction and 2 $\mathrm{cm}$ deep. Squeeze the plunger gently until a small ball (Figure 5) is pushed out, slowly withdraw the needle, then release the pressure and remove the needle.

b) At the same point, extend in the radial form at 80 degrees. Squeeze the plunger gently until a small ball is pushed out, slowly withdraw the needle, then release the pressure and remove the needle. Then, make several small balls in a second circle with a length slightly longer than that of step 1 .

c) At the same point, extend in the radial form at 70 degrees (Figure 6). Squeeze the plunger gently until a small ball is pushed out, slowly withdraw the needle, then release the pressure and remove the needle. Then, make several small balls in a third circle with a length slightly longer than that of step 2 .

d) At the same point, extend in the radial form at 60 degrees. Squeeze the plunger gently until a small ball is pushed out, slowly withdraw the needle, then release the pressure and remove the needle. Then, make several small balls in a fourth circle with a length slightly longer than that of step 3 .

The further the outer injections of the stamen go, the longer the injections. The tips of the stamens should be in the same plane (Figure 7).

5) Taking the design of flower petals as an example (Figures 8-10): Full preparation of A12-201, A12-222, A12-223, A12-224 needles (Figure 4).

a) First, fill the A12-201 needles, one with pink liquid jelly and one with green liquid jelly (1:1 color ratio, with the volume around one circle of the stamen). Along the edge of the stamen, the needle is injected at 60 degrees from the same starting point. At the very bottom of the stamen, the needle slightly curves inward and completes the first petal with the liquid jelly. Continue one by one until the stamen is surrounded to form the first circle of the flower.

b) Take the A12-222 needle with the pink liquid jelly at the same point of 60 degrees into the very bottom of Step 1, which slightly curves inward. Complete the first petal with liquid jelly when the needle is lifted out. Continue one by one until the first round of petals is completed to form the second round. 


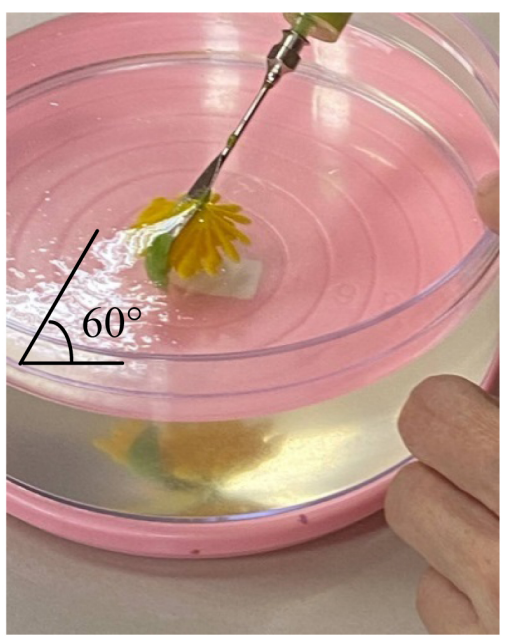

Figure 8. Direct injection at 60 degrees.

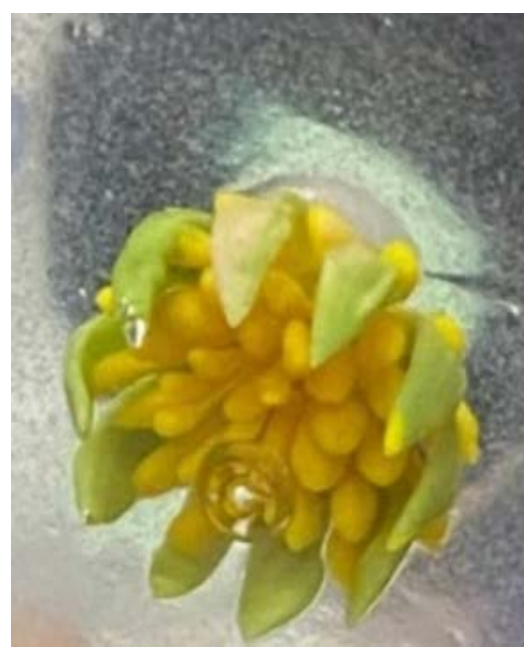

Figure 9. Turn to see the bud.

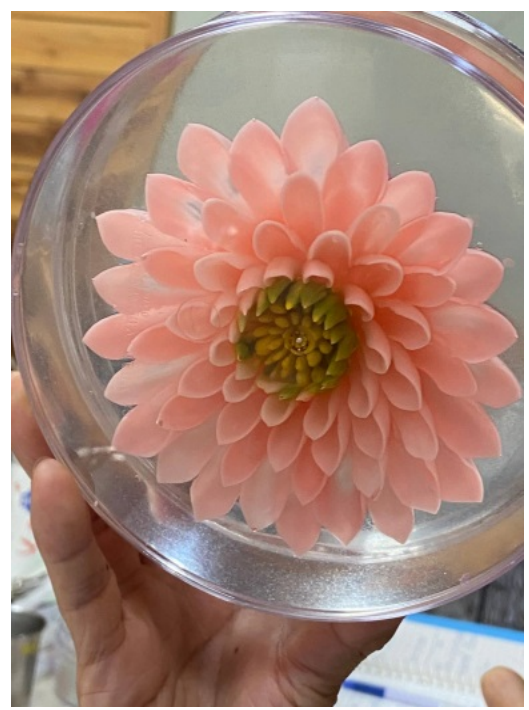

Figure 10. Shape of a floral jelly. 
c) Take the A12-223 needle with the pink liquid jelly at the same point of 60 degrees into the very bottom of Step 2, which slightly curves inward. Complete the first petal with liquid jelly when the needle is lifted out. Continue one by one until the second round of petals is completed to form the third round.

d) Take the A12-224 needle with the pink liquid jelly at the same point of 55 degrees into the very bottom of Step 3 straight. Complete the first petal with liquid jelly when the needle is lifted out. Continue one by one until the third round of petals is completed to form the fourth round.

e) Take the A12-224 needle with the pink liquid jelly at the same point of 50 degrees into the very bottom of Step 4 outward. Complete the first petal with liquid jelly when the needle is lifted out. Continue one by one until the fourth round of petals is completed to form the fifth round.

In order to design a larger flower, the more outer the petals there are, the smaller the radian.

6) Taking the design of large leaf as an example (Figure 11): select the A12208 needle (Figure 4).

a) Take the A12-208 needle with the green liquid jelly, extend the green liquid jelly in $4 \mathrm{~cm}$ and curve left at $1 \mathrm{~cm}$ and 45 degrees. The first leaf is made when the needle is lifted out.

b) At the same point, extend the jelly in $4 \mathrm{~cm}$ and curve right at $1 \mathrm{~cm}$ and 45 degrees. The second leaf is made when the needle is lifted out.

c) At the same point, extend the jelly in $3.5 \mathrm{~cm}$ and curve left at $1.5 \mathrm{~cm}$ and 45 degrees. The third leaf is made when the needle is lifted out.

d) At the same point, extend the jelly in $3.5 \mathrm{~cm}$ and curve right at $1.5 \mathrm{~cm}$ and 45 degrees. The fourth leaf is made when the needle is lifted out.

e) At the same point, extend the jelly in $3 \mathrm{~cm}$ and curve left at $2 \mathrm{~cm}$ and 45 degrees. The fifth leaf is made when the needle is lifted out.

f) At the same point, extend the jelly in $3 \mathrm{~cm}$ and curve right at $2 \mathrm{~cm}$ and 45 degrees. The sixth leaf is made when the needle is lifted out.

g) At the same point, extend the jelly in $3 \mathrm{~cm}$ and curve up at $2 \mathrm{~cm}$ and $45 \mathrm{de}-$ grees. The seventh leaf is made when the needle is lifted out.
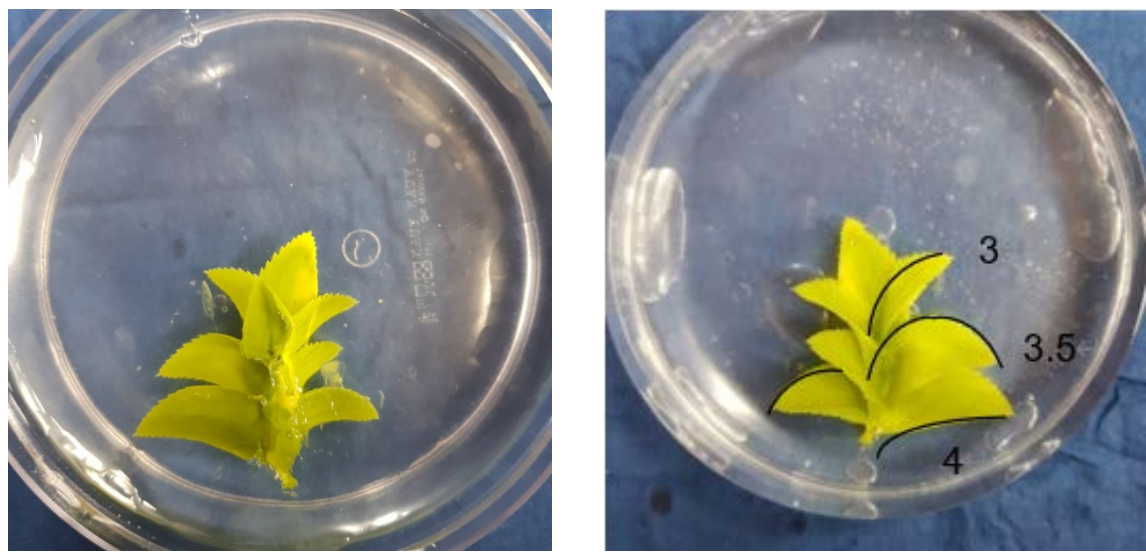

Figure 11. The design of curved shape for leaf. 


\subsection{Result}

Figure 12 shows the record of the various characteristics of the quantitative mathematics introduced to students in the teaching site for the design of floral jelly cakes during this study. Figure 13 shows the front of the floral jelly. The original liquid, which is thick and has different colors, can form the floral art through the application of mathematical knowledge in the teaching design.

It can be seen from the above figures that in addition to different types of tools and angles of injection, the lengths, depths, and radians of injection should also be paid attention to when making flower jelly. The basic knowledge of mathematics and the changes of geometric angles applied with floral jelly and creative ideas are required in this artistic production. Only in this way can we experience the beautiful aesthetics of this visually stunning and dynamic art form.

The achievement of visual stereo effect and aesthetics of geometrical artistic shape depends on the creator's space awareness and whether the cake's location distribution of patterns and the composition's proportion reach the golden ratio. Needless to say, the creator's mathematical literacy is required to feel these elements and carry out the activity. Therefore, mathematical literacy is essential for practitioners in creating desserts in artistic shapes, such as floral jelly cakes.

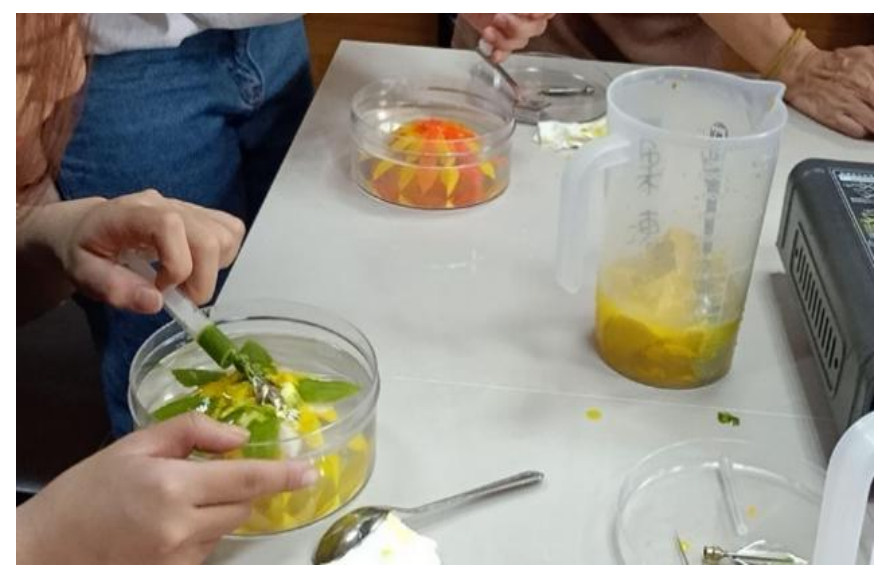

Figure 12. Design moment of students.

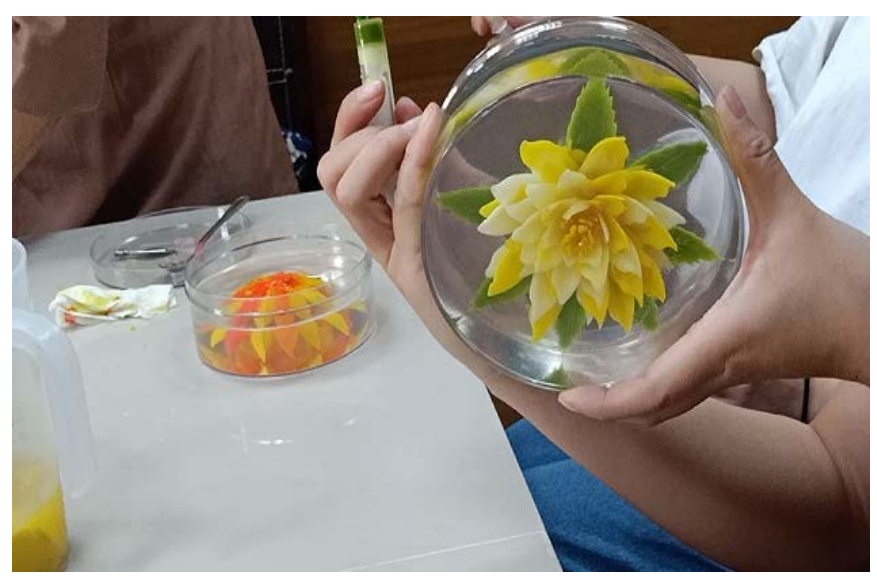

Figure 13. The front of floral jelly made by students. 


\section{Conclusion and Suggestions}

\subsection{Conclusion}

In addition to patience, carefulness, and persistent entrepreneurial spirit, when making a floral jelly cake, practitioners must always be innovative to present unique works to consumers, thus, their artistic and mathematical abilities should be integrated to innovate, change, and improve.

Mathematics comes from life and is everywhere in life. There is certain aesthetic and educational value for students to learn mathematics to master the technical and artistic influence of the floral jelly cake. An important concept of mathematical literacy is to use mathematics to solve problems in life. By understanding these quantitative characteristics, the techniques of this cake can be developed, and the dependency between objects and their variables can be perceived. As Yi and Wang (2007) mentioned, mathematics is an important part of life culture in aesthetic art (Liu, 2016).

This study introduced the mathematical techniques to teach students how to make the floral jelly cake, and the results have an influence on the students' design techniques when making this dynamic structure, thus, the works are highly recognized and pleasing in the market.

\subsection{Research Limitations}

As the finger length and palm force of each person varies, it is inevitable that slight differences remain in the force applied to the liquid jelly injection with different tools, thus, it may be difficult to make all the flower petals and leaves the same. However, the visual and artistic beauty of the floral jelly cake will not be affected.

\subsection{Suggestions}

Follow-up studies are suggested to explore and analyze the different artistic designs for floral jelly cakes before and after students' learning mathematical literacy.

\section{Conflicts of Interest}

The authors declare no conflicts of interest regarding the publication of this paper.

\section{References}

Bian, R. Y. (2018). 3D Floral Jelly with Soul Healing. 8521books.

Chang, C. C. (1988). Basic Design of Stereo Shapes. Grand East Book.

Chen, H. E. (2008). Iconography and Iconology: The Meanings and Interpretations of Visual Arts. Asif Publishing.

Cheng, C. M., Tseng C. W., \& Chao, P. C. (2020). A Study on the Mathematical Literacy of Grade 6 Students. Psychological Testing, 67, 333-353.

Chuang, M. C., \& Chen, C. C. (2004). Exploring the Relationship between the Product Form 
Features and Feature Composition and User's Kansei Evaluation. Journal of Design, 9, 43-56.

Hsiung, H. F., Chu, M., \& Chang, F. (2005). Creativity in the Product Shape Design. Packaging Engineering, 26, 171-173.

Huang, C. A. (1994). On the Universal Meaning and Basic Relation between Art and Mathematics. Journal of Shaanxi Normal University (Philosophy and Social Sciences Edition), 6, 75-83.

Huang, S. W., \& Chou, W. C. (2015). A Study of Shape Fitness between Western Cake and Plate. Journal of Design Environment, 16, 43-57.

Ishibashi, K. (2020). Guilt-Free, Reduced-Sugar Dessert. Kadokawa Taiwan.

Juan, I. M., Tsai, Y. S., Chang, R. H., Lin, K. S., \& Yang, S. S. (1995). Development and Extension of Multiple Tea Products. Taiwan Tea Research Bulletin, 56, 61-72.

Liu, P. H. (2016). Discourse on the Constituent of Literacy for Mathematical Culture in Terms of the Relationship between Mathematics and Culture-Theoretical and Case Analysis. Taiwan Journal of Mathematics Education, 3, 55-83.

Lu, C. F. (1984). Rationale of Shapes. Lionart.

OECD (2018). PISA 2021 Mathematics Framework (Draft) (pp. 1-95). Organization for Economic Cooperation and Development.

Ouyang, F. (1994). Mathematical Art. Chiuchang.

PISA National Center, National Taiwan Normal University (2021). PISA 2022 Mathematical Assessment Framework (pp. 1-5).

Tseng, Z. (2000). Philosophical Identification of Mathematics and Art and Its Significance to Quality Education. Journal of Mathematics Education, 9, 41-44.

Tso, T. Y., \& Lei, K. H. (2018). Design and Development of Mathematical Literacy-Oriented Subject Materials. Journal of Research in Education Sciences, 63, 29-58.

Xiong, Y. X., \& Zhang, H. R. (2015). Fused Ways of Mathematics and Art in Elementary Mathematics Education-Based on Art and Mathematics. Journal of Mathematics Education, 24, 88-91.

Yi, N. H., \& Wang, C. P. (2007). Mathematical Culture from Multiple Perspectives. China Science Publishing.

Yu, C. C. (2016). The Story of Seeing Beauty. National Academy for Educational Research.

Zhang, Y. F. (2007). The Relations between Mathematics and Art. Journal of Liaoning Normal University (Natural Science Edition), 30, 26-27. 